\title{
TIC y la gestión del conocimiento como elementos determinantes del crecimiento de la PyME
}

\author{
ICT and knowledge management determining elements in the \\ growth of SME

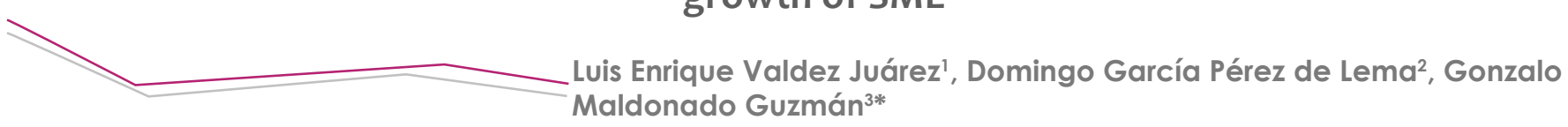

Valdez Juárez, L. E., Pérez de Lema, D. G., Maldonado Guzmán, G. TIC y la gestión del conocimiento como elementos determinantes del crecimiento de la PyME. Investigación y Ciencia de la Universidad Autónoma de Aguascalientes. Número 70: 50-62, enero-abril 2017.

\section{RESUMEN}

Las tecnologías de la información y comunicación (TIC) son un factor determinante para el crecimiento en la pequeña y mediana empresa (PyME) y se han vuelto imprescindibles en las actividades cotidianas de las organizaciones, principalmente en las estrategias de gestión del conocimiento (GC). Además, la actual literatura relaciona a estos indicadores como generadores de crecimiento económico y organizacional, no solo para las grandes, sino también para la PyME. La muestra está conformada por 903 empresas de la región de Murcia, España, y el objetivo principal es analizar la influencia que tienen las TIC sobre la GC y, a su vez, los efectos de las TIC y la GC sobre el crecimiento empresarial. Los hallazgos indican que el uso de las TIC tiene una influencia positiva y significativa en los procesos de GC. La utilización de TIC y GC muestra una influencia positiva en el crecimiento.

\section{ABSTRACT}

The technologies of information and communication (ICT) are a determining factor for competitiveness

Palabras clave: TIC, gestión del conocimiento, crecimiento, PyME.

Keywords: ICT, knowledge management, growth, SME.

Recibido: 22 de marzo de 2016, aceptado: 22 de septiembre de 2016

Departamento de Ciencias Económicas y Administrativas, Instituto Tecnológico de Sonora unidad Guaymas, México.

2 Departamento de Economía Financiera y Contabilidad, Facultad de Ciencias de la Empresa, Universidad Politécnica de Cartagena, España. Departamento de Mercadotecnia, Centro de Ciencias Económicas y Administrativas, Universidad Autónoma de Aguascalientes, México.

* Autor para correspondencia: gmaldona@correo.vaa.mx in the small and medium enterprises (SMEs) and have become indispensable in the daily activities of organizations, mainly in the knowledge management strategies (KM). Literature, in recent times, also relates to these business indicators as generators of economic and organizational growth not only for large companies, but also for SMEs. In this work, the sample consists of 903 companies from the region of Murcia, Spain. The main objective is to analyze the influence that ICT has on the KM and at the same time the effects of ICT and the KM on business growth. The findings indicate that the use of ICT has a positive and significant influence in the processes of KM. The use of ICT and the KM shows a positive influence in the growth of sales, but in the growth of employees there is no significant relationship.

\section{INTRODUCCIÓN}

Actualmente se ha incrementado el interés por el estudio sobre las tecnologías de la información y comunicación (TIC), dado el valor que generan en la gestión de las empresas (Martin y Matlay, 2003; Benítez Amado y Walczuch, 2012; Chen y Huang, 2012). Se ha comprobado que las TIC a través de procesos, técnicas y herramientas mejoran la toma de decisiones empresariales (Bhatt y Grover, 2005; Crawford et al., 2011) y son determinantes para el éxito en una nueva era basada en la economía del conocimiento (Rao, 2012; Stirbu et al., 2015). Además, el conocimiento por su intangibilidad se convierte en ilimitado y dinámico (Nonaka, 2007; Cohendet y Simon, 2008) y debe ser aplicado para que las metas organizacionales se logren oportunamente (Clarke y Rollo, 2001; Chawan y Vasudevan, 2013). Asimismo, la gestión del conocimiento (GC) mejora el manejo de la información (Davenport y Prusak, 1998; Mahesh 
y Suresh, 2004; Davenport, 2013), y conjuntamente con las TIC facilitan la captura de la información, su acopio y el intercambio del conocimiento (Gold et al., 2001; Yew Wong y Aspinwall, 2005; Kaldi et al., 2008; Nfuka y Rusu, 2011), y son una herramienta que favorece la rentabilidad y el crecimiento de la PyME (Rowe, 2013).

Este trabajo aporta a la literatura de las TIC y GC en dos perspectivas. Primero, analiza la influencia de las TIC y de los procesos de GC sobre el crecimiento de la PyME. En la literatura existe un número moderado de estudios empíricos que analizan la influencia que tienen las TIC sobre la GC de una forma genérica (Yew Wong y Aspinwall, 2005; Tseng, 2008; Coakes et al., 2010; Pérez López y Alegre, 2012; Cohen y Olsen, 2015). Pero existe poca evidencia empírica que analice la relación existente entre las variables que miden a la GC (entrenamiento a empleados, políticas y estrategias, adquisición de conocimiento y cultura organizacional). La mayoría de los trabajos que analizan la relación entre el uso de las TIC y la GC han hecho poco énfasis en los beneficios que pueden lograr las PyME (Tseng, 2008; Cohen y Olsen, 2015). Este estudio también identifica los factores más relevantes de la GC que pueden favorecer el crecimiento de la PyME. Segundo, con respecto a la influencia que tiene la GC sobre el crecimiento en la PyME, se ha encontrado que la mayoría de los análisis empíricos se focalizan en el efecto sobre el crecimiento económico (número de clientes, ingresos por explotación, número de ventas y total de activos fijos) (Nummela et al., 2010; Hodson, 2014) y en menor medida se analiza el crecimiento de la organización en función al número de empleados (Lutchen, 201 1; Tarí Guilló y García Fernández, 2011; Andreeva y Kianto, 2012).

Este estudio analiza el incremento de las ventas y de los empleados como componentes del crecimiento empresarial. Además existe una limitada evidencia empírica enfocada en la PyME en esta área del conocimiento (Chawan y Vasudevan, 2013; Piget y Kossaii, 2013). Debido a la importancia del uso de las TIC para el crecimiento de las organizaciones, en el diseño de estrategias empresariales como la implementación de la GC y bajo el contexto actual de la PyME, la investigación tuvo como objetivo principal analizar la influencia que tienen las TIC sobre la GC y los efectos que generan sobre el crecimiento empresarial. Para dar cumplimiento al objetivo de investigación se ha realizado un estudio empírico analizando 903 PyME establecidas en la región de
Murcia, España. El estudio ha sido desarrollado a través de la técnica estadística de regresión lineal por MCO (mínimos cuadrados ordinarios).

\section{Marco teórico y planteamiento de hipótesis}

Las TIC contribuyen en el funcionamiento y mejora de la GC (Desouza, 2003; Tseng, 2008), y más en el entrenamiento de los empleados potenciando su conocimiento (Tunc Bozbura et al., 2007; López Nicolás y Meroño Cerdán, 2011). Por tanto, las TIC a través de herramientas como equipos y programas computacionales promueven el aprendizaje de los empleados y fortalecen el proceso de enseñanzaaprendizaje (Tiwana, 2002; Kaschig et al., 2010), pues estas prácticas contribuyen al entrenamiento del personal de la empresa (Meroño, 2005; Kamel, 2010). Asimismo, las PyME con frecuencia están explotando el capital relacional y estructural a través del uso de las TIC (Kristandl y Bontis, 2007; Ucbasaran et al., 2008; Federico et al., 2009), con la finalidad de aumentar sus competencias y mejorar la eficiencia organizacional (Rojas Mesa, 2006), ya que las TIC apoyan la gestión empresarial a través de herramientas tecnológicas como intranet, correo electrónico, teleconferencias, y otras, con una influencia significativa en la formación del capital humano (Clarysse et al., 2011; Ndubisi y Nwankwo, 2013). Adicionalmente, estudios recientes señalan que las TIC son cruciales en la transferencia y uso del conocimiento, al elevar las competencias del recurso humano (Maier, 2013; Palacios Marqués et al., 2015), y facilitan el desarrollo de sus habilidades, lo que repercute en una mayor intensidad para innovar y mejorar el crecimiento de las PyME (Haile et al., 2013; Hatzikian y Bampasis, 2015). Por tanto, de acuerdo con la información anterior es posible plantear la primera hipótesis de trabajo:

\section{H1: A mayor adopción de las TIC, mayor nivel de entrenamiento de los empleados}

Estudios recientes realizados en la PyME de distintos países de América, Europa, África y Asia, han concluido que el uso de las TIC influye positivamente en algunas prácticas empresariales y en las estrategias para la GC (Wolff y Pett, 2006; Ca'Zorzi, 2011). Además, se ha encontrado que las capacidades de infraestructura tecnológicas son herramientas mediadoras en el establecimiento de las políticas y estrategias empresariales, lo cual permite una mejor gestión del capital humano (Erden et al., 2014). Asimismo, López Nicolás y Meroño Cerdán (2011) y Cohen y Olsen (2015) concluyeron que 
existe una relación significativa entre las políticas de protección y codificación del conocimiento y las TIC. También se afirma que ayudan en el despliegue de las políticas que regulan el conocimiento, la protección de documentos electrónicos, patentes, fórmulas e invenciones (Majors, 2010; Tsui et al., 2014). Por otra parte, existe evidencia empírica que demuestra que las TIC apoyan el establecimiento de políticas empresariales que fortalecen el conocimiento de los empleados, ayudan en las relaciones con los clientes, en el conocimiento de la competencia y en la búsqueda de nuevos mercados (Elder et al., 2013; Spagnol Fedoce et al., 2015). Por ello, en función de la revisión teórica y empírica se plantea la siguiente hipótesis:

\section{H2: A mayor uso de las TIC, mayor influencia en el diseño de políticas y estrategias de GC}

Las TIC son esenciales en el entorno actual de los negocios y se consideran una herramienta capaz de gestionar, adquirir y transmitir el conocimiento estructural (Davenport y Prusak, 1998; Tseng, 2008; Crawford et al., 2011 ). Por ello, las TIC se han convertido en un elemento determinante en el diseño de estrategias que favorecen el crecimiento de la PyME (Alemna y Sam, 2006; Zhang et al., 2008). Identifican las habilidades y determinan el uso eficiente del conocimiento existente y promueven la generación del nuevo conocimiento (Metaxiotis, 2009; Chadha y Saini, 2012). Por ello las TIC son cruciales en la toma de decisiones y en la implementación de un sistema de GC (Guitert et al., 2007; Kaschig et al., 2010). Adicionalmente, Lai et al. (2011) y Wang y Wang (2012) concluyeron que la adquisición de conocimientos a través del uso de las TIC mejora significativamente la innovación tecnológica y el crecimiento de la PyME. López Nicolás y Soto Acosta (2010) encontraron que las TIC tienen una relación significativa en el incremento de la adquisición y creación del conocimiento. Moffett y Hinds (2010) y Pérez López y Alegre (2012) consideraron que las competencias de dichas tecnologías facilitan los procesos de captura de conocimientos. Finalmente, Andreasson (2015) consideró que los directivos deben de invertir en las mismas para mejorar los procesos de adquisición, transferencia y uso del conocimiento, con la finalidad de potenciar el capital intelectual de la organización. Ello lleva a plantear la siguiente hipótesis:

\section{H3: A mayor adopción de las TIC, mayor influencia en la adquisición del conocimiento}

Existe evidencia empírica que demuestra que las TIC y las prácticas de GC contribuyen sustancialmente en el desarrollo de la cultura basada en el trabajo de equipo entre los departamentos, lo cual mejora significativamente el conocimiento de la organización (Edwards, 2005; Kane et al., 2006; Hislop, 2013; Bourke y Crowley, 2015). Con el uso de las TIC se fortalece la GC, ello permite que el personal se comprometa más con la empresa y se refuercen la ética profesional y los principios corporativos (Tseng, 2008; Ordóñez de Pablos y Edvinsson, 2015). La adopción de las tecnologías estudiadas (herramientas tecnológicas como correo electrónico, tutoriales, video digital y las redes) en las PyME tienen una fuerte influencia en la cultura organizacional (Huang y Shih, 2009; Moheno y Vallés, 2009), por medio de ellas es factible transmitir información a los empleados, como valores institucionales, código de ética, filosofía corporativa y actividades de integración, lo cual puede fortalecer la cultura organizacional (Jennex, 2005; Kodama, 2008). La PyME aún está luchando por utilizar herramientas y técnicas novedosas, como los repositorios soportados en sistemas tecnológicos con la finalidad de establecer una cultura que promueva la transferencia del conocimiento (Bechhofer et al., 2010; Coakes et al., 2010). Estudios recientes indican una relación estrecha entre las TIC y la cultura organizacional, factores determinantes en la estrategia exitosa de la GC en la PyME (Malekifar et al., 2014). Se emite la siguiente hipótesis:

\section{H4: A mayor uso de las TIC, mayor influencia en la cultura organizacional}

La GC son prácticas relacionadas con la creación, adquisición, transferencia y uso del conocimiento que se genera al interior y exterior de la organización (Swan et al., 1999; Erickson y Rothberg, 2012), por lo cual las PyME que adopten prácticas de GC tienden a lograr un mayor nivel de crecimiento (Edvardsson y Durst, 2013). Las prácticas de GC por lo regular conducen al logro de un mayor nivel de rentabilidad, elevan los ingresos e inciden en el incremento de los empleados (Lee y Choi, 2003; Hana, 2013). A medida que la empresa va creciendo el número de empleados también lo hace, lo que complica la GC y las actividades 
de los recursos humanos (Cardy y Lengnick Hall, 2011; Mello, 2011), ya que con el crecimiento de los empleados las empresas tienen una mayor dificultad para transferir y controlar el conocimiento (North y Kumta, 2014). Por ello, el incremento del personal tiene una relación significativa con los procesos de GC y con el crecimiento económico (OECD, 2010; Ndubisi y Nwankwo, 2013). A mayor personal es evidente que se genera mayor valor (conocimiento) (Nonaka, 2007; Zalesna, 2014). Recientes estudios empíricos indican que los procesos de GC son una estrategia que permite incrementar las ventas de las PyME (Lee et al., 2008; Tarí Guilló y García Fernández, 2011), y mejoran significativamente su rendimiento económico (Carrillo et al., 2008; Pattanayak, 2014). Las PyME están adoptando a la GC para mejorar no solamente sus procesos, sino también para obtener un mayor crecimiento económico (Constantinescu, 2009; Huang y Shih, 2009), pues se ha demostrado que la GC contribuye significativamente en ellas para obtener más clientes y mayores ventas (Van der Weide, 2012; Martin Rojas et al., 2014). De lo anterior se hace el siguiente planteamiento:

\section{H5: A mayor utilización de la GC, mayor crecimiento de los ingresos}

\section{H6: A mayor utilización de la GC, mayor generación} de empleo

La actual literatura establece que la adopción de las TIC en las empresas se ha realizado con el fin de influir en la productividad (Van Grembergen et al., 2004; Vogelsang, 2010), ignorando su influencia en el capital humano (Newell, 2002) y descuidando un poco el crecimiento organizacional. Existe evidencia empírica que indica que la incorporación de las TIC en la PyME facilita la obtención de resultados económicos y financieros (Rangan y Adner, 2001; Ca'Zorzi, 201 1). En un estudio reciente Ghobakhloo et al. (2011) llegaron a la conclusión de que la adopción de TIC en este dtipo de empresas incide en su nivel de competitividad y crecimiento. De igual manera, Torrent Sellens y Ficapal Cusí (2010) y Benítez Amado y Walczuch (2012) demostraron que las TIC están fuertemente correlacionadas con el crecimiento y la rentabilidad de las empresas. Estos resultados coinciden con los obtenidos en los estudios de Andreeva y Kianto (2012) y Sung y Choi (2012).

El uso estratégico de las TIC también influye en el crecimiento organizacional (número de empleados), cuota de mercado y rendimiento financiero (Moheno y Vallés, 2009; Prieger y Heil, 2009), ya que las TIC mejoran las actividades del individuo y permiten controlar la productividad de cada empleado (Wessels, 2014). Con el desarrollo de nuevas herramientas tecnológicas se ha reducido el número de empleados en las empresas (Van der Weide, 2012; Audretsch y Welfens, 2013). Además, las TIC han provocado una mayor automatización de los procesos productivos, de servicios y comerciales, lo que incide en la disminución de los empleados (Ashford y Hall, 2011 ; Torres Coronas, 2012). Asimismo, estudios recientes señalan que con las TIC y la alineación de una estrategia basada en innovación tecnológica se logran altos niveles de competitividad, se elevan las ventas y se mejoran las habilidades de los empleados de las PyME (Piget y Kossaii, 2013). De lo anterior se hace el siguiente planteamiento:

\section{H7: A mayor utilización de las TIC, mayor crecimiento de los ingresos}

\section{H8: A mayor utilización de las TIC, mayor generación de empleo}

MATERIALES Y MÉTODOS

Para la validación de las hipótesis planteadas en la investigación se desarrolló un estudio empírico en PyME de la región de Murcia, España. La muestra fue establecida a partir de la información del DIRCE (Directorio Central de Empresas) del INE (Instituto Nacional de Estadística), y no se incluyeron empresas de menos de cinco trabajadores. El tamaño muestral fue determinado para lograr que el margen de error máximo para la estimación de una proporción fuese inferior a 0.03 puntos con un nivel de confianza de 95\% (ver Tabla 1). La recolección de datos se realizó durante los meses de abril a julio de 2010, una muestra de 903 empresas (ver Tabla 2). La técnica para recolectar la información fue mediante entrevista personal mediante un cuestionario autoadministrado dirigido al gerente de la empresa (ver Tabla 3).

Las empresas que rechazaron participar en el estudio fueron reemplazadas por una empresa similar de la misma actividad y área geográfica. El sesgo de no respuesta fue analizado de acuerdo con las recomendaciones de Nwachukwu et al. (1997). Las empresas que dieron respuesta en la primera ronda correspondió a $80 \%$ de la muestra, y se compararon con las respuestas de las que respondieron por sustitución (20\% de la muestra). De todas las variables consideradas no surgieron diferencias significativas 
entre los dos grupos utilizando las pruebas $t$ de Student y chi-cuadrada.

\section{Variable dependiente}

En relación con las variables de crecimiento empresarial, se han medido utilizando la variación de las ventas y del número de empleados, las mismas se determinan entre los años 2008 y 2009 y se transforman a través de un logaritmo neperiano. El análisis de las ventas ha sido utilizado para medir el crecimiento empresarial, como por ejemplo en estudios previos de Nummela et al. (2010) y Hodson (2014), y el crecimiento de los empleados en los estudios de Lutchen (2011), Tarí Guilló y García Fernández (2011) y Andreeva y Kianto (2012).

\section{Variable independiente}

En relación con la medición de la variable uso de las TIC se consideraron algunos elementos del modelo de adopción y preparación tecnológica sugerido por Molla y Licker (2005) y Majors (2010) y con las proxys utilizadas por Bascavusoglu Moreau y Colakoglu (2013). Se pidió a los gerentes de las PyME seleccionadas que informaran a través de 10 preguntas si en su empresa disponían o no de ellas (1= Sí y $0=$ No): 1. ¿ Utiliza las nuevas tecnologías para la gestión organizativa de la empresa? , 2. ¿ Se considera bien informado sobre las posibilidades y ventajas de las TIC en la empresa?, 3. ¿Considera necesario que se incentive la formación sobre aplicación TIC en la empresa? , 4. ¿Tiene sU empresa conexión a internet?, 5. ¿Dispone de página web?, 6. ¿̇Dispone de correo electrónico?, 7. ¿ Realiza compras electrónicas usando internet? , 8. ¿ Realiza su empresa teletrabajo? 9. ¿. Realiza venta electrónica usando internet? y 10. ¿ Realiza marketing usando internet?

Para la medición de la GC se utilizaron cuatro dimensiones, medidas en una escala tipo Likert de 5 puntos ( $1=$ desacuerdo, $5=$ total acuerdo): (1) Entrenamiento de los empleados, medido con 4 ítems y adaptada de Liebowitz (1999), Foray y Gault (2003) y Kristandl y Bontis (2007); (2) Políticas y estrategias de la gestión del conocimiento, medido con 9 ítems y adaptada de Tunc Bozbura et al. (2007) y López Nicolás y Meroño Cerdán (2011); (3) Captura del conocimiento externo, medida con 5 ítems y adaptada de Gold et al. (2001), Foray y Gault (2003) y Tunc Bozbura et al. (2007), y (4) Efectos de la cultura organizacional, medida con 4 ítems y adaptada de Foray y Gault (2003) y Tunc Bozbura et al. (2007).

Tabla 1. Diseño de la investigación

\begin{tabular}{|l|l|}
\hline \multicolumn{1}{|c|}{ Características } & \multicolumn{1}{c|}{ Investigación } \\
\hline Población & Pequeñas y Medianas Empresas \\
\hline Área geográfica & Murcia (España) \\
\hline Muestra & PyME de 5 a 250 empleados \\
\hline Método de recolección de información & Entrevistas personales a los gerentes \\
\hline Método de muestreo & Muestreo simple aleatorio \\
\hline Tamaño de la muestra & 903 PyME \\
\hline Error del muestreo & (+/-3\%) error, nivel de confiabilidad de 95\% $(p=q=0.5)$ \\
\hline Trabajo de campo & Abril-julio de 2010 \\
\hline
\end{tabular}

En la tabla se muestra el diseño de la investigación utilizada en el estudio. Elaboración propia.

Tabla 2. Características de la muestra

\begin{tabular}{|l|c|c|}
\hline \multicolumn{1}{|c|}{ Sector } & Número de empresas & Porcentaje total \\
\hline Industrial & 418 & 46.3 \\
\hline Construcción & 186 & 20.6 \\
\hline Servicios & 153 & 16.9 \\
\hline Comercio & 146 & 16.2 \\
\hline Total & 903 & 100 \\
\hline
\end{tabular}

Información de las principales características de la muestra utilizada en el estudio. Elaboración propia. 
Tabla 3. Cuestionario

\begin{tabular}{|c|c|c|c|c|c|}
\hline & & & \multicolumn{3}{|c|}{ Tendencia 2010} \\
\hline Crecimiento empresarial & 2008 & 2009 & + & $=$ & - \\
\hline No. medio de empleados & & & 1 & 2 & 3 \\
\hline \multicolumn{6}{|l|}{ Entrenamiento } \\
\hline $\begin{array}{l}2 \text { Proporciona a sus trabajadores una formación informal relacionada con la } \\
\text { GC }\end{array}$ & 1 & 2 & 2 & 4 & 5 \\
\hline 3 Utiliza prácticas formales de asesoría para sus trabajadores & 1 & 2 & 3 & 4 & 5 \\
\hline \multicolumn{6}{|l|}{ Políticas y estrategias } \\
\hline 3 Tiene procedimientos establecidos de apoyo a la innovación & 1 & 2 & 3 & 4 & 5 \\
\hline 4 Tiene un sistema burocrático & 1 & 2 & 3 & 4 & 5 \\
\hline 5 Tiene acceso a las bases de datos de manera rápida & 1 & 2 & 3 & 4 & 5 \\
\hline 6 Tiene acceso a la información sin ninguna limitación & 1 & 2 & 3 & 4 & 5 \\
\hline \multicolumn{6}{|l|}{7 Tiene un conocimiento claro del sistema industrial de manufactura } \\
\hline \multicolumn{6}{|l|}{ Adquisición del conocimiento } \\
\hline 4 Por favor indique si su empresa... & $\mathrm{TD}$ & $\mathrm{D}$ & $\mathrm{N}$ & A & TA \\
\hline 1 Utiliza el conocimiento obtenido de otras fuentes industriales & 1 & 2 & 3 & 4 & 5 \\
\hline 2 Utiliza el conocimiento obtenido de universidades y centros de investigación & 1 & 2 & 3 & 4 & 5 \\
\hline 1 Alienta a directivos y trabajadores a transferir conocimientos a los empleados & 1 & 2 & 3 & 4 & 5 \\
\hline 2 Tiene un sistema de valores y promoción cultural entre sus trabajadores & 1 & 2 & 3 & 4 & 5 \\
\hline 3 Motiva constantemente a sus empleados a trabajar en equipo & 1 & 2 & 3 & 4 & 5 \\
\hline 4 Alienta a los empleados a desarrollar nuevas ideas y a expresar opiniones & 1 & 2 & 3 & 4 & 5 \\
\hline \multicolumn{6}{|l|}{ Uso de las TIC } \\
\hline 6 Por favor indique si su empresa utiliza las TIC... & Sí= 1 & $\mathrm{No}=2$ & & & \\
\hline \multicolumn{6}{|l|}{1 ¿̇Dispone de página WEB? } \\
\hline \multicolumn{6}{|l|}{2 ¿Dispone de correo electrónico? } \\
\hline \multicolumn{6}{|l|}{3 ¿'Realiza venta electrónica usando internet? } \\
\hline \multicolumn{6}{|l|}{4 ¿Realiza compras electrónicas usando internet? } \\
\hline \multicolumn{6}{|l|}{5 ¿ Realiza marketing usando internet? } \\
\hline \multicolumn{6}{|l|}{7 ¿ Realiza compras electrónicas usando internet? } \\
\hline \multicolumn{6}{|l|}{8 ¿Realiza su empresa teletrabajo? } \\
\hline \multicolumn{6}{|l|}{9 ¿̇ealiza venta electrónica usando internet? } \\
\hline 10 ¿̇Realiza marketing usando internet? & & & & & \\
\hline
\end{tabular}




\section{Variables de control}

El tamaño de la empresa se midió a través del número medio de empleados del año 2009, y la edad de la empresa fue medida por el número de años transcurridos desde la constitución o puesta en marcha de las operaciones de la empresa.

\section{RESULTADOS}

Para validar las hipótesis que se formularon en la investigación y verificar la relación entre las TIC, la GC y el crecimiento empresarial, se utilizó el modelo de regresión lineal por $M C O$. La técnica estadística elegida es la que mejor se adapta al objetivo de la investigación y a la naturaleza de las variables en estudio, con el fin de corroborar con mayor precisión las hipótesis. Los modelos desarrollados son los siguientes:

Modelo 1. Entrenamiento $(G C)_{i=} \beta_{o}+\beta_{1 x}$ TIC (USO) $_{i+} £$ Modelo 2. Políticas y estrategias (GC) ${ }_{i=} \beta_{\circ}+\beta_{1 \times}$ TIC (USO) ${ }_{i+} £$

Modelo 3. Adquisición de conocimiento externo $(\mathrm{GC})_{i=} \beta_{\circ}+\beta_{1 \times}$ TIC (USO) $i+\infty$

Modelo 4. Cultura organizacional (GC) $i=\beta_{\circ}+\beta_{1 \times}$ TIC (USO) ${ }_{i+} £$

Modelo 5. Crecimiento empresarial ${ }_{i}=\beta_{\circ}+\beta_{1 \times} \mathrm{TIC}$ (uso) ${ }_{i+} \beta_{2 \times}$ Tamaño de la empresa ${ }_{+} \beta_{3 \times}$ Edad de la empresa +

Modelo 6. Crecimiento empresarial ${ }_{i}=\beta_{\circ}+\beta_{1 \times}$ GC (Entrenamiento, Políticas, Adquisición del conocimiento y Cultura organizacional) ${ }_{i+} \beta_{2 x}$ Tamaño de la empresa $\beta_{+} \beta_{3 \times}$ Edad de la empresa ${ }_{+} £$

En la Tabla 4 se presentan las relaciones entre las TIC y las cuatro dimensiones que miden la GC. En este sentido se comprueba que existe una relación positiva significativa en todas las dimensiones de GC. En efecto, se aprecia cómo las TIC tienen una relación positiva y significativa sobre el entrenamiento de los empleados $(\beta=0.221, p<0.01)$, al igual que sobre las estrategias y políticas de gestión ( $\beta=0.351, p<0.01$ ), sobre la adquisición del conocimiento externo $(\beta=$ $0.335, p<0.01$ ) y sobre la cultura organizacional $(\beta=$ $0.335, p<0.01$ ). Si se comparan las $\beta$ estandarizadas se puede ver que el mayor impacto de las TIC se produce en la dimensión de estrategias y políticas de las PyME.
Tabla 4. Relación entre variables (TIC y GC)

\begin{tabular}{|l|r|r|r|r|}
\hline \multicolumn{1}{|c|}{ Variables } & $\begin{array}{c}\text { Valor de } \\
\beta\end{array}$ & $\begin{array}{c}\text { Valor } \\
\text { de } \boldsymbol{t}\end{array}$ & $\begin{array}{c}\text { Valor } \\
\text { de } \boldsymbol{f}\end{array}$ & $\begin{array}{c}\boldsymbol{R}^{\mathbf{2}} \\
\text { Ajustado }\end{array}$ \\
\hline $\begin{array}{l}\text { TIC -> } \\
\text { Entrenamiento de } \\
\text { los empleados }\end{array}$ & $0.221^{* * *}$ & 6,813 & $46,424^{* * *}$ & .048 \\
\hline $\begin{array}{l}\text { TIC -> Estrategias } \\
\text { y políticas }\end{array}$ & $0.351^{* * *}$ & 11,257 & $56,726^{* * *}$ & .122 \\
\hline $\begin{array}{l}\text { TIC -> Adquisición } \\
\text { de conocimiento }\end{array}$ & $0.335^{* * *}$ & 10,680 & $44,068^{* * *}$ & .112 \\
\hline $\begin{array}{l}\text { TIC -> Cultura } \\
\text { organizacional }\end{array}$ & $0.169^{* * *}$ & 5,142 & $26,441^{* * *}$ & .029 \\
\hline
\end{tabular}

La tabla muestra el coeficiente estandarizado de cada hipótesis, el valor del estadístico t de Student y el $R^{2}$ ajustado.

$*: p<0.05,{ }^{* *}: p<0.01,{ }^{* * *}: p<0.001$. Elaboración propia.

Tabla 5. Relación entre variables (GC y Crecimiento empresarial)

\begin{tabular}{|l|c|c|}
\hline \multicolumn{1}{|c|}{ Variables } & $\begin{array}{c}\text { Crecimiento } \\
\text { (ingresos) }\end{array}$ & $\begin{array}{c}\text { Crecimiento } \\
\text { (empleados) }\end{array}$ \\
\hline $\begin{array}{l}\text { Gestión del } \\
\text { conocimiento (GC) }\end{array}$ & $\begin{array}{c}0.010^{*} \\
(1,025)\end{array}$ & $\begin{array}{c}0.043 \\
(1,345)\end{array}$ \\
\hline $\begin{array}{l}\text { Tamaño de la } \\
\text { empresa }\end{array}$ & $\begin{array}{c}0.110^{* * *} \\
(2,906)\end{array}$ & $\begin{array}{c}0.369^{* * *} \\
(11,265)\end{array}$ \\
\hline Edad de la empresa & $\begin{array}{c}0.011 \\
(, 993)\end{array}$ & $\begin{array}{c}-0.025 \\
(-, 476)\end{array}$ \\
\hline VIF más alto & 1,082 & 1,088 \\
\hline Valor def & $3,399^{* *}$ & $47,907^{* * *}$ \\
\hline$R^{2}$ Ajustado & 0.012 & 0.139 \\
\hline
\end{tabular}

Debajo de cada coeficiente estandarizado, entre paréntesis, se muestra el valor $t$ de Student.

VIF más alto 1,088

$*: p<0.05, * *: p<0.01, * * *: p<0.001$. Elaboración propia.

En la Tabla 5 se presentan las estimaciones de la relación entre las dimensiones de la GC y el crecimiento de las ventas (H5) y de los empleados (H6). En ella se aprecia que la GC muestra efectos positivos significativos sobre el crecimiento de las ventas $(\beta=0.010, p<0.10)$, pero no sobre el crecimiento de los empleados ( $\beta=0.043)$. Además, el tamaño de la empresa manifiesta una influencia positiva significativa sobre el crecimiento de las ventas $(\beta=0.110, p<0.01)$ y en el crecimiento de los empleados $(\beta=0.369, p<0.01)$. La edad de la empresa no muestra influencia positiva ni significativa sobre el crecimiento en los ingresos $(\beta=0.011)$, al igual que en el crecimiento de los empleados ( $\beta=$ -0.025). Para validar el modelo de regresión lineal de las hipótesis $\mathrm{H} 5$ y H6 se ha contrastado la $R^{2}$ ajustada con valores de (0.012) y (0.139) y valores en $F$ de $\left(3,399^{* * *}\right)$ y $\left(47,907^{* * *}\right)$. Las variables independientes 
del modelo de regresión lineal muestran un valor de inflación de la varianza (VIF) cercano a la unidad de $(1,084)$ y $(1,088)$. Se descarta la presencia de multicolinealidad.

Tabla 6. Relación entre variables (TIC y Crecimiento empresarial)

\begin{tabular}{|l|c|c|}
\hline \multicolumn{1}{|c|}{ Variables } & $\begin{array}{c}\text { Crecimiento } \\
\text { (ingresos) }\end{array}$ & $\begin{array}{c}\text { Crecimiento } \\
\text { (empleados) }\end{array}$ \\
\hline TIC & $0.098^{* * *}$ & 0.051 \\
$(2,559)$ & $(1,478)$ \\
\hline Tamaño de la & $0.095^{* *}$ & $0.368^{* * *}$ \\
empresa & $(2,555)$ & $(11,259)$ \\
\hline Edad de la & 0.018 & -0.010 \\
empresa & $(.249)$ & $(-.392)$ \\
\hline VIF más alto & 1,078 & 1,082 \\
\hline Valor de f & $5,761^{* * *}$ & $48,173^{* * *}$ \\
\hline$R^{2}$ Ajustado & 0.021 & 0.143 \\
\hline
\end{tabular}

Debajo de cada coeficiente estandarizado, entre paréntesis, se muestra el valor $t$ de Student.

VIF más alto 1,082

$*: p<0.05,{ }^{* *}: p<0.01,{ }^{* * *}: p<0.001$. Elaboración propia.

La Tabla 6 presenta los resultados de la relación entre las $\mathrm{TIC}$ y el crecimiento de las ventas $(\mathrm{H} 7)$ y de los empleados (H8). Los resultados indican que las TIC ejercen influencia positiva y significativa sobre el crecimiento de las ventas $(\beta=0.098, p<0.01)$, pero no para el crecimiento de los empleados $(\beta=$ 0.051), con lo cual se confirma que cuando la PyME tiene mayor infraestructura tecnológica se reduce el número de empleados. Además, el tamaño de la empresa influye de modo positivo y significativo sobre el crecimiento de las ventas $(\beta=0.095, p<0.05)$ y sobre el crecimiento de los empleados de la PyME $(\beta=0.368, p<0.01)$. Además, la antigüedad de la empresa no muestra efectos positivos significativos sobre el crecimiento de los ingresos $(\beta=0.018)$, ni con el crecimiento de los empleados $(\beta=-0.010)$. Para validar el modelo de regresión lineal de las hipótesis $\mathrm{H} 7$ y $\mathrm{H} 8$ se contrastó la $R^{2}$ ajustada, con valores de 0.021 y 0.143 , y un valor en $F$ de $5.761^{* * *}$ y $48.173^{* * *}$. Las variables independientes del modelo de regresión lineal muestran un valor de inflación de la varianza (VIF) cercano a la unidad de 1.078 y 1.082, y se elimina la presencia de multicolinealidad.

DISCUSIÓN

Los resultados obtenidos indican que las TIC son un factor clave en la mejora de las actividades empresariales y para la GC, lo que permite elevar los índices de rentabilidad y crecimiento de las PyME (Majors, 2010; Cohen y Olsen, 2015). Aunque no existe un método y/o técnica apropiada para la implementación de un sistema de GC (Tseng, 2008), queda confirmado que para lograr un mayor rendimiento empresarial es vital el uso de las TIC (Darroch, 2005; Chawan y Vasudevan, 2013). Estos resultados demuestran que su uso mejora sustancialmente: 1) las competencias del capital humano, 2) la comunicación de las estrategias y políticas, y 3) la promoción de la cultura organizacional (Tseng, 2008; Battistella et al., 2016). Es un excelente medio para adquirir más y mejor conocimiento en la PyME, lo que la convierte en una fuente de ventaja competitiva (Yew Wong y Aspinwall, 2005; Andersen, 2015).

El resultado más notable que se encontró en el estudio es que el uso de las TIC influyen significativamente en las políticas y estrategias de la GC de la PyME, resultados que están alineados con la literatura (Allameh y Abbas, 2010; Clarysse et al., 2011; Zawawi et al., 2011). También tiene un efecto positivo y significativo en la adquisición del conocimiento, ya que son el medio más importante en la actualidad para generar y adquirir conocimiento en las empresas (Tseng, 2008; Davenport, 2013), y son la fuente más importante para la generación de nuevo conocimiento en la PyME (Moffett y Hinds, 2010; Pérez López y Alegre, 2012). Con respecto a la relación entre las TIC y el entrenamiento de los empleados, los resultados obtenidos son similares a los obtenidos por Bolívar Ramos et al. (2012) y Glaub et al. (2014), ya que las mismas mejoran las competencias del capital humano y la formación de los empleados (Tunc Bozbura, 2007).

La influencia que tienen las TIC sobre la cultura organizacional en los resultados obtenidos fueron semejantes a los obtenidos por Alavi et al. (2005) y Zerwas (2014) al afirmar que su uso mejora la cultura organizacional y fortalece los valores y principios en la empresa. Adicionalmente, se ha encontrado que contribuyen positivamente en el crecimiento de los ingresos de las PyME, resultados que tienen similitud con la literatura, con ello se corrobora que existe una correlación significativa entre ambas variables (Ca'Zorzi, 2011). La GC permite un crecimiento de las ventas en las empresas y es un elemento determinante para mejorar sus procesos, aumentar la eficiencia y obtener mayores ventas, lo que conduce a un mayor crecimiento empresarial (Constantinescu, 2009; Huang y Shih, 2009; Young y Milton, 2011 ). Finalmente, no se encontró evidencia empírica para la relación entre el uso de las TIC y 


\section{INVESTIGAGIÓก Y CIECEIA DE LA UNIVERSIDAD AUTÓNOMA DE RGUASCALIERTES}

Figura 1. Se estudia la influencia de las TIC en el funcionamiento de las PyME. Imagen obtenida del sitio electrónico Servicio de atención al cliente.com.

el crecimiento de los empleados, lo que corrobora la influencia negativa que expone la literatura. No se encontró evidencia empírica para la relación entre las prácticas de GC y el crecimiento de los empleados en la PyME.

\section{CONCLUSIONES}

De acuerdo con los resultados obtenidos es posible concluir que: 1) el uso de las TIC es crucial en el desarrollo exitoso de un proceso de GC, lo que permite fortalecer las prácticas empresariales como el despliegue de las estrategias y políticas, la captura del conocimiento y de la información, la capacitación de los empleados y la mejora de la cultura organizacional dentro de la empresa pequeña y mediana; 2) tiene incidencia en el crecimiento económico de la PyME y 3) la misma deberá seguir fortaleciendo la infraestructura tecnológica y sus procesos de GC para mejorar el crecimiento empresarial. Este estudio tiene una serie de implicaciones que pueden conducir

al fortalecimiento de la dirección estratégica y al desarrollo empresarial de la PyME. De esta forma es esencial que los dueños de las empresas consideren su uso como un soporte en los procesos de GC para obtener mejores resultados y una fuerte ventaja competitiva (Ca'Zorzi, 2011; Jneid y Tannous, 2012; Rebic y Sarenac, 2014). Los directivos deben considerar dentro de sus objetivos el uso de las TIC como un medio para proteger y potenciar el conocimiento del capital humano, lo que se convierte en un reto y en una oportunidad para la PyME en su camino hacia el éxito (Tseng, 2008; Cohen y Olsen, 2015).

La investigación presenta también algunas limitaciones, como es el caso de la muestra utilizada, ya que solamente se consideró la opinión de los directivos $y / o$ gerentes, se puede contemplar a futuro información de empleados y clientes. La información recopilada puede conducir al sesgo de los resultados, debido a que los datos fueron obtenidos de percepciones subjetivas emitidas por los directivos de la PyME.

En segundo lugar, la muestra ha sido enfocada en las PyME, puede extenderse a otros sectores productivos y a los grandes corporativos para poder comparar los resultados.

Otra limitación la constituyen las escalas utilizadas para la medición de la GC, pues se consideraron únicamente variables reflectivas con adaptaciones de escalas de otros investigadores, por lo que sería aceptable la utilización de variables de tipo reflectivo-formativo. Además, en la medida para el uso de las TIC se consideraron solamente variables de tipo nominal con preguntas dicotómicas. 
LITERATURA CITADA

- ALAVI, M. et al. An empirical examination of the influence of organizational culture on knowledge management practices. Journal of Management Information Systems, 22(3): 191-224, 2005

- ALEMNA, A. A. y SAM, J. Critical issues in information and communication technologies for rural development in Ghana. Information Development, 22 (4): 236-241, 2006.

- ALLAMEH, S. M. y ABBAS, S. K. The relationship between knowledge management practices and innovation level in organizations: case study of sub-companies of selected corporations in the city of Esfahan. Journal of Business Case Studies, 6(1): 1-15, 2010.

- ANDERSEN, T. K. Employees' involuntary non-use of ICT influenced by power differences: a case study with the grounded theory approach. IJIKM, 10: 117-143, 2015.

- ANDREASSON, K. (Ed.). Digital Divides: The new challenges and opportunities of e-inclusion. New York, NY, US: CRC Press, 2015.

- ANDREEVA, T. y KIANTO, A. Does knowledge management really matter? Linking knowledge management practices, competitiveness and economic performance. Journal of Knowledge Management, 16(4): 617-636, 2012.

- ASHFORD, N. A. y HALL, R. P. Technology, Globalization, and Sustainable Development: Transforming the Industrial State. London, UK: Yale University Press, 2011.

- AUDRETSCH, D. B. y WELFENS, P. J. J. The New Economy and Economic Growth in Europe and the US. Berlin, Germany: Springer Berlin Heidelberg, 2013.

- BASCAVUSOGLU MOREAU, E. y COLAKOGLU, M. Impact of SME policies on innovation capabilities: The Turkish case. 9. In I. Hakan Yetkiner et al. (Eds.), Industrial Dynamics, Innovation Policy, and Economic Growth through Technological Advancements (pp. 159-184). IGI Global, 2013.

- BATTISTELLA, C. et al. Inter-organisational technology/knowledge transfer: a framework from critical literature review. The Journal of Technology Transfer, 41 (5): 1195-1234, 2016.

- BENÍTEZ AMADO, J. y WALCZUCH, R. M. Information technology, the organizational capability of proactive corporate environmental strategy and firm performance: a resourcebased analysis. European Journal of Information Systems, 21 (6): 664-679, 2012.

- BHATT, G. D. y GROVER, V. Types of information technology capabilities and their role in competitive advantage: An empirical study. Journal of Management Information Systems, 22(2): 253-277, 2005.

- bolívar RAMOS, M. T. et al. Technological distinctive competencies and organizational learning: Effects on organizational innovation to improve firm performance. Journal of Engineering and Technology Management, 29(3): 331-357, 2012.
- BOURKE, J. y CROWLEY, F. The role of hrm and ict complementarities in firm innovation: evidence from transition economies. International Journal of Innovation Management 19(5): 50-54, 2015

- CARDY, R. L. y LENGNICK HALL, M. L. Will they stay or will they go? Exploring a customer-oriented approach to employee retention. Journal of Business and Psychology, 26(2): 213-217, 2011.

- CARRILLO, F. J. et al. Knowledge-based capital in building regional innovation capacity. Journal of Knowledge Management, 12(5): 121-136, 2008.

- CA'ZORZI, A. Adoption and use of information systems and technology in manufacturing small and medium-sized enterprises. European Journal of Information Systems, 12, 127141,2011

- CHADHA, S. K. y SAINI, R. Key enablers in the implementation of KM practices: an empirical study of software SMEs in North India. The IUP Journal of Knowledge Management, X(4): 59-85, 2012.

- CHAWAN, A. N. y VASUDEVAN, H. Modeling knowledge management barriers in the Indian manufacturing SMEs using ISM approach. The IUP Journal of Knowledge Management, XI(4): 36-52, 2013

- CHEN, Y. Y. y HUANG, H. L. Knowledge management fit and its implications for business performance: A profile deviation analysis. Knowledge-based systems, 27, 262-270, 2012.

- ClARKE, T. y ROLLO, C. Corporate initiatives in knowledge management. Education+ Training, 43(4/5): 206-214, 2001.

- CLARYSSE, B. et al. Entrepreneurial origin, technological knowledge, and the growth of spin-off companies. Journal of Management Studies, 48(6): 1420-1442, 2011.

- COAKES, E. et al. Knowledge management, strategy, and technology: a global snapshot. Journal of Enterprise Information Management, 23(3): 282-304, 2010.

- COHEN, J. F. y OLSEN, K. Knowledge management capabilities and firm performance: A test of universalistic, contingency and complementarity perspectives. Expert Systems with Applications, 42(3): 1178-1188, 2015.

- COHENDET, P. y SIMON, L. Knowledge intensive firms, communities and creative cities 1. In A. AMIN y J. ROBERTS (Eds.), Community, Economic Creativity, and Organization (pp. 227-253). UK: Oxford University Press, 2008.

- CONSTANTINESCU, M. Knowledge management: focus on innovation and labor productivity in a knowledge-based economy. The IUP Journal of Knowledge Management, 7(1): 7-33, 2009

- CRAWFORD, J. et al. The human resource's influence in shaping IT competence. Industrial Management \& Data Systems, 111 (2): 164-183, 2011. 
- DARROCH, J. Knowledge management, innovation and firm performance. Journal of Knowledge Management, 9(3): 101115,2005

- DAVENPORT, T. H. Process Innovation: Reengineering Work through Information Technology. Boston, MA, US: Harvard Business Press, 2013

- DAVENPORT, T. H. y PRUSAK, L. Working Knowledge: How Organizations Manage what they know. Boston, MA, US: Harvard Business Press, 1998.

- DESOUZA, K. C. Facilitating tacit knowledge exchange. Communications of the ACM, 46(6): 85-88, 2003.

- EDVARDSSON, I. R. y DURST, S. The benefits of knowledge management in small and medium-sized enterprises. ProcediaSocial and Behavioral Sciences, 81, 351-354, 2013.

- EDWARDS, A. Let's get beyond community and practice: the many meanings of learning by participating. The Curriculum Journal, 16(1): 49-65, 2005.

- ELDER, L. et al. Connecting ICTs to Development: The IDRC Experience. New York, NY, US: Anthem Press, 2013.

- ERDEN, Z. et al. Knowledge-flows and firm performance. Journal of Business Research, 67(1): 2777-2785, 2014.

- ERICKSON, G. S. y ROTHBERG, H. Intelligence in Action: Strategically Managing Knowledge Assets. New York, NY, US: Palgrave Macmillan, 2012.

- FEDERICO, J. et al. Factores determinantes del crecimiento en empresas jóvenes. Evidencias de una comparación internacional. En Nuevas empresas en América Latina: factores que favorecen su rápido crecimiento. 1 (pp. 21-31). España: Universitat Autònoma de Barcelona, 2009.

- FORAY,D.yGAULT, F. Measurementofknowledgemanagement practices. In Measuring Knowledge Management in the Business Sector. Canada: OECD/Minister of Industry, 2003.

- GHOBAKHLOO, M. et al. Information technology adoption in Small and Medium-sized Enterprises; An appraisal of two decades literature. Interdisciplinary Journal of Research in Business, 1 (7): 53-80, 2011

- GLAUB, M. E. et al. Increasing personal initiative in small business managers or owners leads to entrepreneurial success: a theory-based controlled randomized field intervention for evidence-basedmanagement. Academy of Management Learning \& Education, 13(3): 354-379, 2014.

- GOLD, A. H. et al. Knowledge management: An organizational capabilities perspective. Journal of Management Information Systems, 18(1): 185-214, 2001.

- GUITERT, M. et al. Competencias TIC y trabajo en equipo en entornos virtuales. RUSC. Revista de Universidad y Sociedad del Conocimiento, 4(1): 1-14, 2007.

- HAILE, G. A. et al. The impact of globalization and technnology transfer on manufacturing employment and skills in Ethiopia. IZA Discussion Paper, 7820 1-25, 2013.
- HANA, U. Competitive advantage achievement through innovation and knowledge. Journal of Competitiveness, 5(1): 82-96, 2013.

- HATZIKIAN, Y. y BAMPASIS, E. Exploring the relationship of innovation intensity, knowledge production and productivity in greek SMEs before the eruption of debt crisis. Journal of the Knowledge Economy, 1, 1-25, 2015.

- HISLOP, D. Knowledge Management in Organizations: A Critical Introduction, UK: Oxford University Press, 2013.

- HODSON, R. Workers' learnings and corporate economic structure. New York, NY, US: Academic Press, 2014.

- HUANG, P.S. y SHIH, L. H. Effective environmental management through environmental knowledge management. International Journal of Environmental Science and Technology, 6(1): 35-50, 2009.

- JENNEX, M. E. Knowledge management. Wiley Encyclopedia of Management, 2005.

- JNEID, M. y TANNOUS, A. Strategic Management and Information Evaluation Challenges Facing Entrepreneurs of SMEs in ICT. Paper presented at the 6th European Conference on Information Management and Evaluation, 2012.

- KALDI, A. et al. KMS adoption in organizations. Paper presented at the Industrial Engineering and Engineering Management, IEEM 2008. IEEE International Conference, 2008.

- KAMEL, S. E-Strategies for Technological Diffusion and Adoption: National ICT Approaches for Socioeconomic Development. New York, NY, US: IGI Global, 2010.

- KANE, $\mathrm{H}$. et al. Knowledge management methodologies. The Electronic Journal of Knowledge Management, 4(2): 141-152, 2006.

- $\quad$ KASCHIG, A. et al. Knowledge maturing activities and practices fostering organisational learning: Results of an empirical study. Sustaining TEL: From Innovation to Learning and Practice, 1: 151166, 2010.

- KODAMA, M. New Knowledge Creation Through ICT Dynamic Capability Creating Knowledge Communities Using Broadband. Charlotte, NC, US: IAP, 2008.

- KRISTANDL, G. y BONTIS, N. Constructing a definition for intangibles using the resource based view of the firm. Management decision, 45(9): 1510-1524, 2007.

- LAI, M. C. et al. Designing a knowledge-based system for benchmarking: A DEA approach. Knowledge-based systems, 24(5): 662-671, 2011.

- LEE, C. L. et al. The impact of knowledge management enablers on non-financial performance in small and medium enterprises. International Journal of Technology Management, 43(1-3): 266283, 2008.

- $\quad \mathrm{LEE}, \mathrm{H}$. y CHOI, B. Knowledge management enablers, processes, and organizational performance: An integrative view and empirical examination. Journal of Management Information Systems, 20(1): 179-228, 2003. 
- LIEBOWITZ, J. (Ed.). Knowledge Management Handbook. New York, NY,US: CRC Press, 1999.

- LÓPEZ NICOLÁS, C. y MEROÑO CERDÁN, Á. L. Strategic knowledge management, innovation and performance. International Journal of Information Management, 31 (6): 502$509,2011$.

- LÓPEZ NICOLÁS, C. y SOTO ACOSTA, P. Analyzing ICT adoption and use effects on knowledge creation: An empirical investigation in SMEs. International Journal of Information Management, 30(6): 521-528, 2010.

- LUTCHEN, M. D. Managing IT as a Business: A Survival Guide for CEOs. Ney York, NY, US: John Wiley \& Sons, 2011.

- MAHESH, K. y SURESH, J. K. What is the K in KM technology? The Electronic Journal of Knowledge Management, 2(2): 11-22, 2004.

- MAIER, R. Knowledge Management Systems: Information and Communication Technologies for Knowledge Management. Berlin, Germany: Springer Berlin Heidelberg, 2013.

- MAJORS, I. ICT and knowledge management models for promotion of SME's competitiveness. The International Journal of Technology, Knowledge and Society, 6(3): 173-184, 2010.

- MALEKIFAR, S. et al. Organizational culture, IT Competence, and supply chain agility in Small and Medium-Size Enterprises. Global Business and Organizational Excellence, 33(6): 69-75, 2014.

- MARTIN, L. M. y MATLAY, H. Innovative use of the Internet in established small firms: the impact of knowledge management and organisational learning in accessing new opportunities. Qualitative Market Research: An International Journal, 6(1): 18-26, 2003.

- MARTIN ROJAS, R. et al. Knowledge-based organization in tourism industry. Engineering Economics, 25(1): 82-93, 2014.

- MELLO, J. A. Strategic management of human resources. New York, NY, US: South-Western/Cengage Learning, 2011

- MEROÑo, A. L. El correo electrónico en las Pymes para la comunicación y gestión del conocimiento. Universia Business Review-Actualidad Económica, 1: 71-79, 2005.

- METAXIOTIS, K. (Ed.). Intelligent Information Systems and Knowledge Management for Energy: Applications for Decision Support, Usage, and Environmental Protection. Hershey, PA, US: IGI Global (International Publisher of Progressive Academic Research), 2009.

- MOFFETT, S. y HINDS, A. Assessing the impact of KM on organisational practice: applying the MeCTIP model to UK organizations. Electronic Journal of Knowledge Management, 8(1): 103-117, 2010

- MOHENO, G. A. y VALLÉS, R. S. Explorando el uso de las TI en la relación entre la innovación y los procesos de creación del conocimiento en las pequeñas y medianas empresas. Presentado en el XIII Congreso de Ingeniería de Organización, 2009.

- MOLLA, A. y LICKER, P. S. E-commerce adoption in developing countries: a model and instrument. Information \& Management, 42(6): 877-899, 2005.

- NDUBISI, N. O. y NWANKWO, S. Enterprise Development in SMEs and Entrepreneurial Firms: Dynamic Processes. New York, NY, US: Business Science Reference, 2013.

- NEWELL, S. Creating the healthy organization: Well-being, diversity and ethics at work. London: Cengage Learning EMEA, 2002.

- NFUKA, E. N. y RUSU, L. The effect of critical success factors on IT governance performance. Industrial Management \& Data Systems, $111(9)$ : 1418-1448, 2011.

- NONAKA, I. The Knowledge-Creating Company. Harvard Business Review, 2007

- NORTH, K. y KUMTA, G. Knowledge Management: Value Creation Through Organizational Learning. Switzerland: Springer International Publishing, 2014.

- NUMMELA, N. et al. Knowledge and experience in the internationalization of knowledge-intensive firms. The Theory and Practice of Entrepreneurship: Frontiers in European Entrepreneurship Research, 1: 101-110, 2010.

- NWACHUKWU, S. L. et al. Ethics and social responsibility in marketing: an examination of the ethical evaluation of advertising strategies. Journal of Business Research, 39(2): 107118, 1997.

- OECD (Organization for Economic Cooperation and Development). Education Today 2010: The OECD Perspective. OECD Publishing, 2010.

- ORDÓÑEZ DE PABLOS, P. y EDVINSSON, L. (Eds.). Intellectual capital in organizations: non-financial reports and accounts. London, UK: Taylor y Francis, 2015.

- PALACIOS MARQUÉS, D. et al. New Information and Communication Technologies for Knowledge Management in Organizations: 5th Global Innovation and Knowledge Academy Conference, GIKA 2015, Valencia, Spain, July 14-16, 2015, Proceedings: Springer International Publishing, 2015.

- PATTANAYAK, B. Human Resource Management. New York, NY, US: PHI Learning, 2014.

- PÉREZ LÓPEZ, S. y ALEGRE, J. Information technology competency, knowledge processes and firm performance. Industrial Management \& Data Systems, 112(4): 644-662, 2012.

- $\quad$ PIGET, P. y KOSSAÏ, M. The relationship between information and communication technology use and firm performance in developing countries: a case study of electrical and electronic goods manufacturing SMEs in Tunisia. African Development Review, 25(3): 330-343, 2013

- PRIEGER, J. E. y HEIL, D. The microeconomic impacts of e-business on the economy. In School of Public Policy Working Papers. Paper 11. Pepperdine University, 2009.

- $\quad$ RANGAN, S. y ADNER, R. Profitable Growth in Internet-Related Business: Strategy Tales and Truths. Working Papers-INSEAD R \& D., 2001. 
- RAO, M. (Ed.). Knowledge management tools and techniques. Practitioners and Experts Evaluate KM Solutions. London: Routledge, 2012.

- REBIC, M. y SARENAC, N. Technological progress as a generator of economic growth and development. Journal of Economic and Social Studies, 4(2): 73-99, 2014.

- ROJAS MESA, Y. De la gestión de información a la gestión del conocimiento. Acimed, 14(1): 10-20, 2006.

- ROWE, W. B. Principles of Modern Grinding Technology. London: Elsevier Science, 2013.

- SPAGNOL FEDOCE, R. et al. Knowledge management as a competitive advantage to the brazilian MVAS ecosystem. Journal of Technology Management \& Innovation, 10(2): 1-8, 2015.

- STIRBU, O. A. et al. Theoretical approach regarding the competitiveness of SMEs. International Journal of Advanced Research, 3(6): 1057-1063, 2015.

- SUNG, S. Y. y CHOI, J. N. Effects of team knowledge management on the creativity and financial performance of organizational teams. Organizational Behavior and Human Decision Processes, 118(1): 4-13, 2012.

- SWAN, J. et al. Knowledge management and innovation: networks and networking. Journal of Knowledge Management, 3(4): 262-275, 1999.

- TARÍ GUILLÓ, J. J. y GARCÍA FERNÁNDEZ, M. ¿Puede la gestión del conocimiento influir en los resultados empresariales? Cuadernos de Gestión, 13(1): 151-176, 2011.

- TIWANA, A. The Knowledge Management Toolkit: Orchestrating IT, Strategy, and Knowledge Platforms. India: Pearson Education India, 2002.

- TORRENT SELLENS, J. y FICAPAL CUSÍ, P. TIC, co-innovación y productividad empresarial: evidencia empírica para Cataluña y comparación internacional de resultados. Revista de Economía Mundial, 1 (26): 203-233, 2010.

- TORRES CORONAS, T. Social E-Enterprise: Value Creation through ICT. New York, NY, US: IGI Global, 2012.

- TSENG, S. The effects of information technology on knowledge management systems. Expert Systems with Applications, 35(12): 150-160, 2008.

- TSUI, E. et al. Knowledge-based extraction of intellectual capital-related information from unstructured data. Expert Systems with Applications: An International Journal, 41(4): 1315-1325, 2014.

- TUNC BOZBURA, F. Knowledge management practices in Turkish SMEs. Journal of Enterprise, Information Management, 20(2): 209-221, 2007.

- TUNC BOzBURA, F. et al. Prioritization of human capital measurement indicators using fuzzy AHP. Expert Systems with Applications, 32(4): 1100-1112, 2007.
- UCBASARAN, D. et al. Opportunity identification and pursuit: does an entrepreneur's human capital matter? Small Business Economics, 30(2): 153-173, 2008.

- VAN DER WEIDE, T. P. A Digital (R) evolution to the Information Age. Globalization, Technology Diffusion and Gender Disparity, $1,1-14,2012$.

- VAN GREMBERGEN, W. et al. Structures, processes and relational mechanisms for IT governance: theories and practices. 1. In W. VAN GREMBERGEN (Ed.), Strategies for information technology governance (pp. 1-36). IGI Global, 2004.

- VOGELSANG, M. Digitalization in Open Economies: Theory and Policy Implications. New York, NY, US: Physica-Verlag, 2010.

- WANG, Z. y WANG, N. Knowledge sharing, innovation and firm performance. Expert Systems with Applications, 39(10): 88998908, 2012.

- WESSELS, B. Exploring Social Change: Process and Context. New Yoky, NY, US: Palgrave Macmillan, 2014.

- WOLFF, J. A. y PETT, T. L. Small-firm performance: modeling the role of product and process improvements*. Journal of Small Business Management, 44(2): 268-284, 2006.

- YEW WONG, K. y ASPINWALL, E. An empirical study of the important factors for knowledge-management adoption in the SME sector. Journal of Knowledge Management, 9(3): 64-82, 2005.

- YOUNG, T. y MILTON, N. Knowledge management for sales and marketing: a practitioner's guide. Chandos Publishing, 2011.

- ZALESNA, A. An induction process for new employees and intellectual capital in SMEs: a proposed theoretical link. Proceedings of the 6th European Conference on Intellectual Capital, ECIC, 2014.

- ZAWAWI, A. A. et al. The study of barrier factors in knowledge sharing: a case study in public university. Management Science and Engineering, 5(1): 59-70, 2011.

- ZERWAS, D. Organizational culture and absorptive capacity: the meaning for SMEs. London: Springer Fachmedien Wiesbaden, 2014.

- $\quad$ ZHANG, P. et al. Two types of attitudes in ICT acceptance and use. International Journal of Human-Computer Interaction, 24(7): 628-648, 2008.

\section{De páginas electrónicas}

- BECHHOFER, S. et al. Research objects: Towards exchange and reuse of digital knowledge. In Nature Precedings, The Future of the Web for Collaborative Science (FWCS 2010), Raleigh, NC, US, 26 April, 2010. doi: 10.1038/npre.2010.4626.1

- SERVICIO de Atención al Cliente.com [Imagen]. Recuperado de http://serviciodeatencionalcliente.com/ nuevos-enfoques-empresas-tic/ 